\title{
Wichtige Änderungen bei der AHV per 1. Januar 2009
}

Andreas Friedli

Korrespondenz:

Andreas Friedli

Ingenieur FH, dipl. Treuhandexperte Jürg Dahinden

Treuhand \& Steuerpraxis

FMH Treuhand Services

Thormannmätteliweg 29

CH-3004 Bern

Tel. 0313023804

Fax 0313028140

andreas.friedli@fmhtreuhand.ch

www.fmhtreuhand.ch
Mit der Inkraftsetzung des Bundesgesetzes über die Familienzulagen per 1. Januar 2009 ergeben sich für die Arztpraxis ganz wichtige Änderungen. Der Artikel zeigt auf, welche Anpassungen der Arzt vornehmen und mit welchen neuen Belastungen er rechnen muss.

\section{Neuregelung der Familienzulagen}

Per 1. Januar 2009 hat jedes Kind, für das der Bezüger oder die Bezügerin von Familienzulagen aufkommt, im Minimum einen Anspruch auf Kinderzulagen von monatlich Fr. 200.- bis zum vollendeten 16. Lebensjahr und eine Ausbildungszulage von Fr. 250.- bis zum Abschluss der Ausbildung (längstens jedoch bis zum vollendeten 25. Altersjahr). Der Gesetzgeber hat es den Kantonen überlassen, weitergehende Zulagen und höhere Ansätze festzulegen - davon haben einige Gebrauch gemacht (Tab. 1).

\section{Tabelle 1}

Arten und Ansätze der Zulagen nach den kantonalen Gesetzen.

\begin{tabular}{|c|c|c|c|c|}
\hline Kanton & $\begin{array}{l}\text { Ansatz je K } \\
\text { Kinderzulage }\end{array}$ & $\begin{array}{l}\text { und Monat } \\
\text { Ausbildungszulage }\end{array}$ & Geburtszulage & Adoptionszulage \\
\hline $\mathrm{ZH}$ & 200 & 250 & - & - \\
\hline $\mathrm{BE}^{1}$ & 230 & 290 & - & - \\
\hline LU & $200 / 210^{2}$ & 250 & 1000 & 1000 \\
\hline UR & 200 & 250 & 1000 & 1000 \\
\hline sZ & 200 & 250 & 1000 & - \\
\hline ow & 200 & 250 & - & - \\
\hline NW & 240 & 270 & - & - \\
\hline GL & 200 & 250 & - & - \\
\hline$Z G^{*}$ & 300 & $300 / 350^{3}$ & - & - \\
\hline $\mathrm{FR}^{4}$ & $230 / 250^{5}$ & $290 / 310^{5}$ & 1500 & 1500 \\
\hline so & 200 & 250 & - & - \\
\hline BS & 200 & 250 & - & - \\
\hline $\mathrm{BL}^{*}$ & 200 & 250 & - & - \\
\hline SH & 200 & 250 & - & - \\
\hline $\mathrm{AR}^{*}$ & 200 & 250 & - & - \\
\hline Al & 200 & 250 & - & - \\
\hline$S^{*}{ }^{*}$ & 200 & 250 & - & - \\
\hline GR & 220 & 270 & - & - \\
\hline$A G^{*}$ & 200 & 250 & - & - \\
\hline $\mathrm{TG}^{*}$ & 200 & 250 & - & - \\
\hline $\mathrm{Tl}^{*}$ & 200 & 250 & - & - \\
\hline$V^{4}$ & $200 / 370^{5}$ & $250 / 420^{5}$ & 1500 & 1500 \\
\hline vs & $275 / 375^{5}$ & $425 / 525^{5}$ & $2000^{6}$ & $2000^{6}$ \\
\hline $\mathrm{NE}^{4}$ & $200 / 250^{5}$ & $280 / 330^{5}$ & 1200 & 1200 \\
\hline GE & $200^{7} / 300^{5}$ & $250 / 350^{5}$ & $1000 / 2000^{5}$ & $1000 / 2000^{5}$ \\
\hline JU & 250 & 300 & 850 & 850 \\
\hline
\end{tabular}

* Kantonale Vorlage bei Redaktionsschluss noch nicht defintiv verabschiedet.

1 Die einzelnen FAK können höhere und weitere Zulagen vorsehen.

2 Der erste Ansatz gilt für Kinder bis zu 12 Jahren, der zweite für Kinder über 12 Jahren.

3 Der erste Ansatz gilt für Kinder bis zum 18. Altersjahr, der zweite für Kinder ab dem 18. Altersjahr.

4 Die einzelnen FAK können höhere Zulagen vorsehen.

5 Der erste Ansatz gilt für die ersten beiden Kinder, der zweite für jedes weitere Kind.

6 Bei Mehrfachgeburten oder -adoptionen 3000 Franken pro Kind.

7 Kinderzulagen für erwerbsunfähige Kinder von 16 bis 20 Jahren 250 Franken, ab dem dritten Kind 350 Franken. 


\section{Kinderzulagen bei Teilzeitarbeit}

Die bisherigen Teilzulagen - wovon vor allem Medizinische Praxisassistentinnen aufgrund von Teilzeitpensen betroffen waren - fallen weg. Ab einem Minimallohn von Fr. 553.- pro Monat (Fr. 6630.- pro Jahr) besteht ein Anspruch auf eine Kinder- oder Ausbildungszulage. Nach Gesetz haben auch die Ehegatten, die im Betrieb ohne Lohn mitarbeiten, Anspruch auf die Familienzulagen, dies jedoch nur bis zu einer Steuereinkommensgrenze von Fr. 41040.-. Klar gesetzlich geregelt worden sind neu auch das Doppelbezugsverbot und die Anspruchskonkurrenz, wenn mehrere Personen für das gleiche Kind Anspruch auf Familienzulagen haben.

\section{Selbständige neu anspruchsberechtigt, aber nicht überall!}

Leider konnte per Gesetzeseinführung keine einheitliche Regelung der Zulagenberechtigung für selbständig Erwerbende getroffen werden. Nebst den bisherigen Kantonen, in welchen Selbständige bereits Zulagen erhielten, sind sie ab 1. Januar 2009 in Basel-Stadt, Glarus und Bern anspruchsberechtigt. Als einziger Kanton hat Graubünden auf das bisher gültige Bezugsrecht verzichtet.

\section{Tabelle 2}

Neuer Ansatz für den FAK-Beitrag.

\begin{tabular}{ll} 
Kanton & Beitragssatz \\
\hline AR & $0,9 \%$ \\
\hline BL & $1,5 \%$ (bis Fr. 126000.-) \\
\hline BS & $0,7 \%$ (bis Fr. 126000.-) \\
\hline BE & $1,1 \%$ (bis Fr. 126000.-) \\
\hline GL & $0,1 \%$ \\
\hline SH & $1,0 \%$ \\
\hline
\end{tabular}

Bemerkungen

Geschäftssitz im Kanton

Geschäftssitz im Kanton

Geschäftssitz im Kanton

Geschäftssitz im Kanton

Geschäftssitz im Kanton

\section{Neue Finanzierungsregelung}

Bisher entrichteten nur Arbeitgeber von der AHVLohnsumme Beiträge an die Familienausgleichskassen. Um die zusätzlichen Beiträge für selbständig Erwerbende zu finanzieren, werden neu die Inhaber von Betrieben ohne Angestellte auch zur Kasse gebeten. Der FAK-Beitrag wird bei diesen auf ihrem selbständigen, AHV-pflichtigen Einkommen ermittelt und zusammen mit der AHV-Festsetzung in Rechnung gestellt. Die folgenden Ansätze werden ihnen gemäss Tabelle 2 neu in Rechnung gestellt.

Entlastet werden mit der Neuregelung die Beitragsätze auf der AHV-Lohnsumme für die Angestellten, die zum Beispiel im Kanton Bern von 1,6 auf $1,1 \%$ abgesenkt wird. Per Saldo muss aber die Arztpraxis mit einer Mehrbelastung von Fr. 450.- bis 1350.- rechnen!

\section{Welche Auswirkungen ergeben sich für die Arztpraxis?}

Der Arzt/die Ärztin sollte per Januar 2009 die Situation bei allen Angestellten inklusive den Ehegatten überprüfen. Sie müssen die nach Kanton festgesetzten Kinder- oder Ausbildungszulagen ihren Angestellten monatlich vergüten und die entsprechenden Anpassungen vornehmen. Die Ärzteschaft sollte damit rechnen, dass aufgrund der geänderten Finanzierungsgrundlagen ihre Praxen mit neuen Mehrauslagen konfrontiert werden.

Aus den geschilderten Gründen erhält die definitive AHV-Festsetzung bei der Ärztin oder dem Arzt eine weitere Abgabekomponente und ihre Überprüfung und das Einleiten von Rechtsschritten in der gesetzten Frist bei Fehlerhaftigkeit zusätzliche Bedeutung. Konsequenz: Nicht nur Steuerentscheide sondern auch AHV-Entscheide sollte der Arzt von seinem Treuhänder auf deren Korrektheit überprüfen lassen! 